\title{
A Grounded Theory Analysis of COVID-19 Information and Resources Relayed Through University Webpages: Implications for a More Inclusive Community
}

\section{Dr. Sreyoshi Bhaduri, McGraw Hill}

Dr. Sreyoshi Bhaduri is an Engineering Educator and People Researcher. She currently heads Global People Research and Analytics at McGraw Hill, where she leads research leveraging employee data to generate data-driven insights for decisions impacting organizational Culture and Talent. Her research interests include assessing the impact and effectiveness of inclusion initiatives as well as employing innovative, ethical and inclusive mixed-methods research approaches using AI to uncover insights about the 21 st century workforce. Sreyoshi is passionate about improving belonging among women in STEM and Engineering. She was recently elected as Senator at the Society of Women Engineers - a not for profit organization with over 42,000 global members and the world's largest advocate and catalyst for change for women in engineering and technology. She is also a member of the Society for Industrial and Organizational Psychology. Learn more about her work and get in touch at www.ThatStatsGirl.com.

\section{Dr. Lilianny Virguez, University of Florida}

Lilianny Virguez is a Instructional Assistant Professor at the Engineering Education Department at University of Florida. She holds a Masters' degree in Management Systems Engineering and a Ph.D. in Engineering Education from Virginia Tech. She has work experience in telecommunications engineering and teaches undergraduate engineering courses such as engineering design and elements of electrical engineering. Her research interests include the intersection of core non-cognitive skills and engineering students' success.

\section{Dr. Debarati Basu, University of North Carolina at Charlotte}

Dr. Debarati Basu is an Assistant Teaching Professor in the Department of Software and Information Systems in the College of Computing and Informatics at the University of North Carolina at Charlotte. She earned her Ph.D. in Engineering Education from Virginia Tech (VT) in 2018. She received her bachelor's and masters in Computer Science and Engineering. Her research is at the intersection of Engineering Education and Computing Education Research and focuses on Cyberlearning and engagement, Curriculum development, assessment and evaluation, and experiential learning including undergraduate research. She has been teaching in active learning environments, such as project-based learning and flipped classrooms. She aims to bring in engineering education research into practice.

\section{Dr. Michelle Soledad, Ohio State University}

Michelle Soledad is a Lecturer in the Department of Engineering Education at The Ohio State University. She holds degrees in Electrical Engineering (BS, ME) from the Ateneo de Davao University (ADDU) in Davao City, Philippines, and in Engineering Education (PhD) from Virginia Tech. Her research interests include learning experiences in fundamental engineering courses and data-informed reflective practice. Michelle's professional experience includes roles in industry and academia, having worked as a software engineer, project lead and manager before becoming Assistant Professor and Department Chair for Electrical Engineering at the Ateneo de Davao University in the Philippines. 


\title{
A Grounded Theory Analysis of COVID-19 Information and Resources Relayed Through University web pages: Implications for a more inclusive community
}

\begin{abstract}
This qualitative study investigates web pages documenting COVID-19 responses from 28 universities across the United States. Using grounded theory methodology, we inductively developed a model of universities' response to the pandemic. Four types of strategies were identified from the data and a theoretical model was developed describing (a) causal conditions that underlie the strategies for response to the pandemic, (b) the context that influenced the strategies adopted by the universities, (c) intervening conditions due to the pandemic that influenced strategy development, and (d) potential recommendations to make universities' responses more inclusive. This research has implications for improving the experience of the communities a university serves, including faculty development, especially for newer faculty who are joining the universities remotely and interacting with new colleagues only through the virtual mediums. Finally, this paper will be of use to engineering educators and administrators as they seek to improve inclusion and belonging within faculty at universities.
\end{abstract}

Keywords: Engineering Education faculty; grounded theory; website; covid-19; inclusion; wellbeing

\section{Introduction}

The sudden outbreak of the COVID-19 pandemic had a significant impact on the higher education system, including universities, colleges, and other educational institutions. Most of these institutions previously worked predominantly in an on-campus environment but had to rapidly transition to an online environment due to lockdowns and other pandemic-related restrictions. Due to these unprecedented changes, universities had to provide necessary information and resources to support their multiple stakeholders, which typically include students, faculty, staff, and their families, in a timely manner. One of the most obvious ways that all universities communicated the changes was through their websites. Every university had at least one web page dedicated to communicating different facets of COVID-19 related information and resources. Different universities took different approaches to serve their communities.

An important stakeholder that the university web pages serve are engineering educators and faculty members, similar to us, the authors of this paper. We are international engineering educators in the United States, who are in the early phases of our careers, and identify as women of color. As universities switched to the online format during the Spring 2020 semester, we, like many of our colleagues, had to adapt to new and unusual circumstances as well as make significant adjustments to our professional and personal lives. At the onset of the pandemic, we created a support group to share our experiences as we navigated these unprecedented circumstances. Through the course of the months following the sudden shift in learning 
environments, we continued to reflect and record our personal challenges of both navigating this unique time as well as meeting our evolving responsibilities as engineering educators in this oftchanging landscape.

Reflecting on our own insights, we realized that due to our identities highlighted above, we also tended to experience the pandemic in both similar and different ways in comparison with our other colleagues here in the United States. We also realized that we relied heavily on information from our respective university websites as we navigated these unfamiliar times. We found that we frequented the university websites to learn about answers to questions related to a host of concerns. These concerns included those related to immigration changes and travel advisories (for example, when will we be able to visit our country and our families?), logistics and planning (for example, when will we have to engage with students in-person?), or even personal or health related (for example, when will I have access to the vaccine?). Thus, we quickly understood that these COVID-19 websites were helping address concerns not only of students and their parents but also other stakeholders from diverse backgrounds, who just like us were facing a different set of challenges and looking for answers. We decided to study different university web pages to understand how universities were communicating information and changes through the pandemic and collate strategies that administrators were sharing to help their specific learning communities face the challenges brought on by the ongoing pandemic.

\section{Purpose}

The purpose of this paper is to present topics emphasized by universities on their websites as they helped their learning communities be better equipped to take on the pandemic. We do so by using a Grounded Theory analysis conducted on the landing pages of the college of engineering's COVID-19 sites. We explored the content of the main web page containing COVID-19 related information and resources of 28 universities - both public and private, located in the United States. Our approach is unique because it helped us inductively develop a theory of universities' response to the pandemic, through constant comparison of content data from different universities while limiting any preconceived notions or biases. Ultimately, our analysis is grounded in the views of the universities on taking on the pandemic, as expressed through their content in the COVID-19 related web pages.

\section{Grounded Theory Methodology}

Grounded theory is a systematic qualitative research methodology initially developed as a way to merge qualitative and quantitative approaches in social science research [1], [2] and is considered as an emerging methodology in engineering education research [3]. This methodology enables researchers to build theory from data through constant comparison, identifying analytical codes and categories from data, and then using these categories to generate theories and theoretical models [4]. Our approach thus takes advantage of features of the constructivist approach of Charmaz [4], as well as that of Corbin and Strauss [5] who place a greater emphasis on developing a theoretical model with causal mechanisms.

Given that the purpose of this qualitative research was to describe the "how" question related to universities' response to the pandemic and develop a conceptual model that illustrates the way COVID-19 information and resources were relayed through universities web pages, grounded 
theory methodology was considered an appropriate approach. Ultimately a causal mechanism explaining the strategies that universities used to respond to the COVID-19 pandemic was established. Figure 1 illustrates the iterative steps followed in the study: the steps for analysis are in gray boxes and the steps for quality control are in the boxes with dashed border.

Figure 1. Steps followed in Grounded Theory

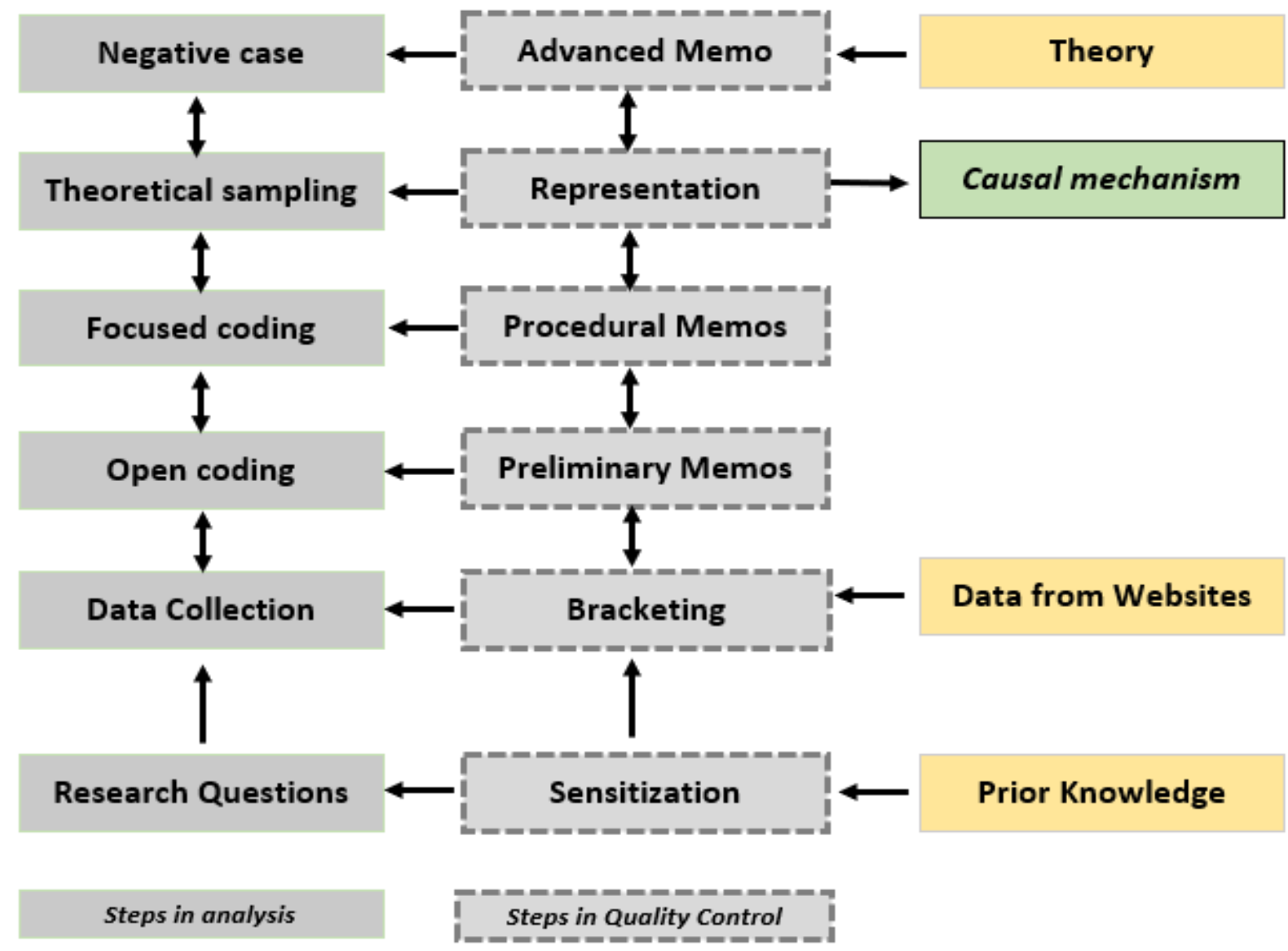

Grounded theory methodology enables researchers "recognizing prior knowledge and theoretical preconceptions and subjecting them to rigorous scrutiny" [5, p. 402]. This is recognizing the value of researchers' perspectives and prior knowledge to enhance their theoretical sensitivity as well as the study potential to contribute to this pre-existing knowledge. Sensitizing concepts provide a foundation for the development of the research questions and the theory generation in the study. In fact, social researchers tend to consider sensitizing concepts as a starting point for a qualitative study [6]. Sensitizing concepts are ideas that serve as the foundation for the overall research problem, as defined by Charmaz [7]. These background ideas "offer ways of seeing, organizing, and understanding experience" [7, p. 259], providing the starting point for analysis.

Thus, our study was initiated by recognizing what we knew about our own universities' response to the pandemic. Accordingly, we sought to learn more about how this process worked in other institutions. Our initial open research question was "How did universities in the U.S respond to the COVID-19 pandemic based on information and resources relayed through their web pages?' We strove to maintain empathetic neutrality in collecting and analyzing data while also 
addressing and acknowledging our personal experiences through the pandemic. In this regard, we maintained individual and group memos, bracketed our reactions and emotions, and took copious notes as we partook in the data collection and analysis process. Creswell [8] defines bracketing as a way in which the researcher can separate their own experiences from what is being studied while memoing consists of recording reflective notes about what the researcher is learning from the data. More information about the steps in analysis and parallel steps to ensure quality of research and insights is presented in the section on Data Analysis.

\section{Researcher Positionality}

All authors except the last were involved in data analysis. The first author has three years of industry experience working as a People Research \& Analytics Manager. The other authors are currently junior faculty primarily teaching courses in colleges of engineering and computer science. All of us are international engineering education researchers in the United States and identify as women of color. Our diverse experiences throughout the COVID-19 pandemic, as individuals and as international women of color in engineering education in the U.S. living through the pandemic, have led us to think more deeply about the importance of inclusive content on university websites since these serve as first point of contact for a diverse community of stakeholders.

Additionally, all members of the research team identify as pragmatists. Our approach to data collection and analysis was thus informed by a pragmatic worldview. Pragmatism places emphasis on shared meanings and joint actions [9]. The pragmatic perspective also emphasizes the role of the researchers in making the choices about what is important, and those choices inevitably involve aspects of our personal history, social background, and cultural assumptions $[9$, p. 69]. Pragmatism aims to investigate the factors that "affect whether the knowledge we gain can be transferred to other settings" [10, p. 4]. Researchers, such as Morgan [12], have elaborated on the use of pragmatism with grounded theory. We followed opinions from grounded theorists related to the mixing of grounded theory traditions and selective adoption of their components [12]. We framed our study primarily within the pragmatic theoretical perspective [13] but also considered Charmaz's constructivist paradigm especially when it came to iterative memoing and bracketing.

\section{Data Collection}

As in other qualitative studies, the data for grounded theory can come from various sources, including web pages. Pioneering Grounded theory researchers have opined that "anything that may shed light on questions under study" can constitute data [13, p. 5]. The average sample size for grounded theory is typically twenty-five data points [14]. In this study, data was collected from the main web pages displaying COVID-19 related information of the first 28 (13 private and 15 public) universities ranked in the 2021 Best Engineering Schools in the United States [15]. Our selection followed a purposive sampling strategy, whereby the researcher "selects the units to be observed on the basis of [a] judgment about which ones will be the most useful" [16, p. 189]. This sampling of universities is similar to the approach carried out by Bhaduri and Roy [17] when selecting higher education institutions for their research. After selecting the 28 
universities to be included in the study, we visited each of the college or school of engineering web pages where the information related to COVID-19 was displayed. The content of each of the web pages were then transcribed verbatim into a single excel file organized by columns.

\section{Data Analysis}

The analytic process was based on immersion in the data of the university web pages and subsequent repeated sorting, coding, and comparisons between the web pages of different universities and between the first three authors of the research team. Analysis began with open coding, which is the process of generating early-stage labels for words, phrases or other elements of the data [14]. The language and phrases used in the websites guided the development of code and category labels. These open codes were iteratively compared and contrasted yielding broader categories of the data (Figure 3, below). Further, memos were written to record the progression of the codes as well as observations and thoughts of the researchers during the coding process (self-reflective memos). Open coding was followed by focused coding, which was the process of selecting "what seem to be the most useful initial codes" and when "we compare data with data and then data with codes" [19, p. 42]. We began with more than a hundred open codes and ended up with 10 focused codes, selected open codes and all the focused codes are depicted in Figure 2 below.

Figure 2. Screenshot of Miro Board, with selected sample open codes, grouped into focused codes. Miro Board is a virtual tool which was used to interactively whiteboard the coding process 


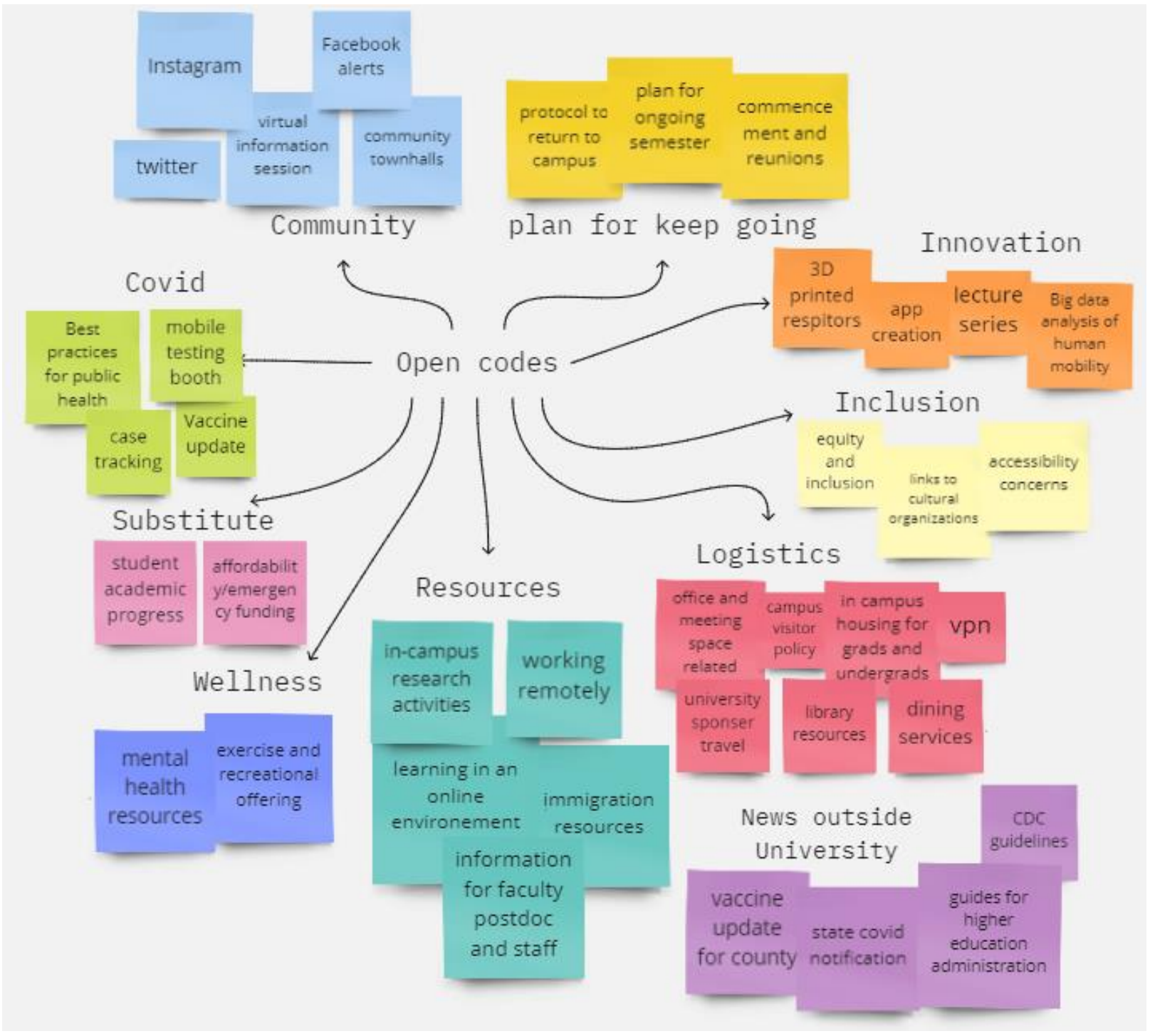

\section{Results}

As described in previous sections, we used Grounded Theory since we wanted to uncover how universities responded to the pandemic by developing categories and themes inductively from the content of the COVID-19 web pages rather than imposing any predetermined classifications, assumptions, hypotheses, etc. on the narrative (similar to the exemplar Grounded theory research conducted by Morrow and Smith [19] who also cite Glaser [20] to validate this approach to analysis).

The theoretical model developed suggests that for the phenomenon under consideration (i.e., universities responding to the pandemic through sharing information with stakeholders through their websites), the causal mechanism (i.e., providing support and creating community) succeed for a set of causal conditions (starting off from a disruption and addressing different priorities) which shape the phenomenon. These causal conditions impacted the causal mechanism, which is identified as universities providing support to its stakeholders, while also attempting to create a sense of community and togetherness as the pandemic storm is weathered. 
Intervening conditions are typically "broad, general conditions" that influence the strategies adopted by the participants in Grounded Theory research [20]. The context (i.e., ongoing COVID-19 pandemic) as well as intervening conditions (e.g., national and state policies and guidelines, university leadership, university resources/values/culture) influence the specific strategies adopted (e.g., providing information and alerts to the community, addressing logistics, promoting wellness, and leveraging the pandemic to showcase innovation and research) that lead to an outcome (i.e., becoming increasingly inclusive). Our Grounded Theory model for university responses to the COVID-19 pandemic is diagrammatically represented in Figure 3.

Figure 3. Grounded Theory Model for university responses to the COVID-19 pandemic

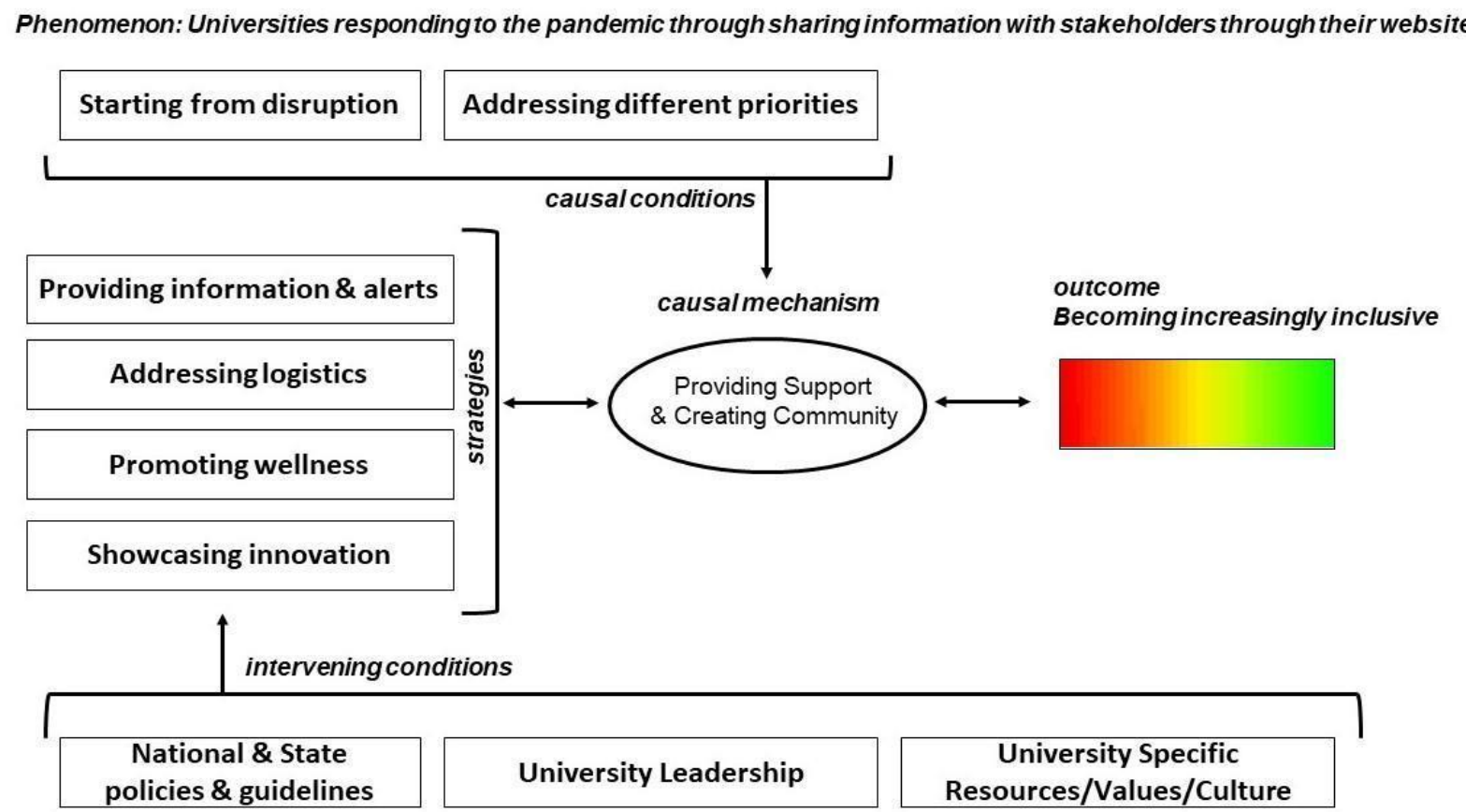

Our analysis uncovered 4 main strategies that universities employed. These were:

1. Providing information and alerts: This was the top strategy employed by the universities. All universities used the COVID-19 related web page to convey information about the pandemic, lockdowns, etc. Universities also used the space to send out alerts to their community related to COVID-19 contexts such as vaccine availability, state and local news, etc. Although every university provided information and alerts on COVID-19 pandemic, only some segregated information according to the need of different stakeholders including researchers, teachers, staff, and postdocs, and only some included immigration information to help international students and faculty navigate the difficult time.

2. Addressing logistics: Around half of the universities used the web pages to address logistics related information. These included resources for faculty and students alike on which buildings were still open, recommendations for use of laboratory spaces, booking of meeting rooms and conference rooms and guidelines for gathering indoors. 
3. Promoting wellness - both physical and mental: Around a third of the universities used the web pages to promote wellness, both physical and mental. Some universities provided links to indoor exercises, while others promoted mindfulness and encouraged mental wellbeing.

4. Showcasing innovation: Only a tenth of the universities used the web pages to showcase innovation or spotlight the work that was being done by their faculty (including research or teaching or service). This seemed like a missed opportunity for faculty development that could easily be adopted by more institutions.

The frequency table below provides an overview of the strategies incorporated by the different universities. The universities have been intentionally de-identified and are listed randomly, since the purpose is not to call out any university, but rather provide an opportunity for all institutions to learn from each other's online messaging to better their inclusion with respect to using web pages for faculty development related tasks. 


\begin{tabular}{|c|c|c|c|c|}
\hline $\begin{array}{c}\text { University \# } \\
\text { (De-identified) }\end{array}$ & $\begin{array}{l}\text { Providing information and } \\
\text { alerts }\end{array}$ & Addressing logistics & Promoting wellness & $\begin{array}{l}\text { Showcasing } \\
\text { innovation }\end{array}$ \\
\hline 1 & $\mathrm{x}$ & $\mathrm{x}$ & & \\
\hline 2 & $x$ & & $\mathrm{x}$ & \\
\hline 3 & $x$ & & & \\
\hline 4 & $x$ & & $x$ & \\
\hline 5 & $x$ & & $\mathrm{x}$ & $x$ \\
\hline 6 & $\mathrm{x}$ & & & \\
\hline 7 & $x$ & $x$ & $\mathrm{x}$ & \\
\hline 8 & $x$ & & & \\
\hline 9 & $x$ & $x$ & & $x$ \\
\hline 10 & $x$ & $x$ & $x$ & \\
\hline 11 & $x$ & & & \\
\hline 12 & $\mathrm{x}$ & $\mathrm{x}$ & & \\
\hline 13 & $x$ & $x$ & & \\
\hline 14 & $x$ & & & \\
\hline 15 & $x$ & $x$ & $x$ & $x$ \\
\hline 16 & $\mathrm{x}$ & $\mathrm{x}$ & & \\
\hline 17 & $\mathrm{x}$ & $x$ & & \\
\hline 18 & $x$ & $x$ & $\mathrm{x}$ & \\
\hline 19 & $x$ & $x$ & & \\
\hline 20 & $\mathrm{x}$ & & & \\
\hline 21 & $x$ & & & \\
\hline 22 & $\mathrm{x}$ & & & \\
\hline 23 & $x$ & $x$ & & \\
\hline 24 & $x$ & & & \\
\hline 25 & $\mathrm{x}$ & $x$ & & \\
\hline 26 & $x$ & & & \\
\hline 27 & $x$ & & $\mathrm{x}$ & \\
\hline 28 & $x$ & $x$ & $x$ & \\
\hline
\end{tabular}

Table 1. Frequency table depicting strategies employed by the different universities as observed on their web pages related to COVID-19

\section{Implications for Faculty Development}

Based on the strategies that emerged from the data and how prevalent these strategies were implemented in university websites, there remain untapped opportunities for some institutions to promote and engage employees in faculty development. The five following recommendations flow directly from the strategies that we identified in the model from the previous section (Figure 3 ), including best practices that some institutions were able to integrate into their websites.

1. Supporting New Faculty - The pandemic has not been kind to new faculty who have been forced to join their new jobs virtually. These individuals are probably not aware of the university culture, resources, logistics and the like, and are possibly going through virtual-only orientations and interactions. For example, for new international faculty, 
additional critical information will include immigration-related processes, updates, and implications of changing laws. Since they do not have ongoing relationships with the university, a recommendation is for COVID-19 web pages to link to resources that will help better support new faculty, whether domestic or international.

2. Highlighting Opportunities for Professional Development - Job requirements for faculty have dramatically metamorphosed as a result of the pandemic. Easy access to resources and logistics might be helpful for both new and existing faculty. These may include spotlights to resources developed by centers of teaching excellence (e.g., CITT at University of Florida), pairing graduate teaching assistants with faculty (e.g., conducted by Virginia Tech's Academy for Graduate Teaching Assistants), as well as workshops delivered by peer faculty (e.g., held at University of North Carolina at Charlotte).

3. Encouraging Mental Health Awareness - As a result of the pandemic, faculty have reported increased stress levels, higher degrees of burn out, unfamiliarity and lowered engagement in working from home. Specifically for new international faculty, some unique added stresses included issues related to immigration laws and the prevailing climate regarding immigration and immigrants. Similarly, there is typically a lot of stress associated with evaluation for new faculty, and this may have increased as a result of the pandemic. If the expectations are reduced or clearly articulated it may help new faculty during the pandemic. Mental health wellness and development can be thought of as an under-explored area of faculty development which can be addressed by universities as part of their response. This is an opportunity for universities to provide mental health resources to help faculty better themselves, such as the resources, tips and advice for mental wellness highlighted by Texas A\&M on their COVID-19 response page.

4. Incentivizing Innovation - Faculty across the globe have experienced being demotivated and feeling bogged down by increased course load and teaching commitments which have shifted dramatically due to the pandemic. Additionally, the pandemic has exacerbated the already siloed engineering departments and reduced opportunities to be interdisciplinary. Highlighting across the university opportunities to network, as well as incentivizing innovative research and teaching by faculty will likely foster increased engagement and motivation.

5. Being Increasingly Inclusive in Who is Spotlighted - The pandemic has resulted in higher rates of dropout among women across the globe. Increasing inclusion and helping better sense of belonging might help faculty, especially those from minority communities, persist. While earlier it was possibly easier to recognize or highlight achievements of faculty which in turn had a positive impact on motivation and job satisfaction, the pandemic makes it difficult to celebrate the small wins. Being cognizant of who is celebrated and acknowledged is critical in current times, especially remembering to do so for individuals from diverse backgrounds. 


\section{Looking Ahead}

Websites have an important role to play in academia and education, and not only in times of an unprecedented pandemic, but always. It is extremely critical that the content of these websites serve its community in an inclusive way. We acknowledge that there are different factors that could have affected the creation of COVID-related content on university websites, which include time constraint, dynamic and oft-changing nature of COVID policies, institutional resources, values, and priorities. The purpose of this research was not to evaluate one website versus the other, nor highlight which university did a better job in conveying information. Rather the goal was to come up with an understanding of different strategies taken by various universities for serving their communities. We believe this insight will help inform and improve inclusive content on university websites when communicating information to a diverse population during an unprecedented event, just as they did during the COVID-19 pandemic. Inclusive university website content can enrich the academic community by creating support and bettering faculty and student experiences thus improving the work and learning environment of diverse stakeholders.

Our paper is U.S. centric and a key recommendation looking ahead would be to expand this model to comprise global institutions. Additionally, usability and accessibility are two important aspects of how websites are used, and our paper does not factor in either considerations. Rather, our primary focus was on analyzing the content and not web design. However, usability and accessibility are both integral to how content is consumed as well as who is able to consume information, and thus must also be looked into. For example, our notes from our bracketing exercises while collecting the data reveal that we found some web pages to feel more like directories and collation of links, rather than an interactive and engaging medium to support their communities. It is hoped that this research will help identify opportunities to inclusively support faculty development, even in times of a pandemic.

\section{Acknowledgement}

The authors extend their heartfelt gratitude to Dr. Elizabeth Creamer, Professor Emerita, Educational Research at Virginia Tech and author, Advancing Grounded Theory with Mixed Methods (Routledge, forthcoming, 2021) for her time, detailed feedback, and for sharing her expertise with the authors whilst they drafted this paper.

\section{References}

[1] G. Glaser Barney and L. Strauss Anselm, The discovery of grounded theory: strategies for qualitative research. Chicago, IL: Aldine, 1967.

[2] K. Charmaz, Constructing Grounded Theory. SAGE, 2014.

[3] J. M. Case and G. Light, "Emerging Research Methodologies in Engineering Education Research," J. Eng. Educ., vol. 100, no. 1, pp. 186-210, Jan. 2011.

[4] J. M. Corbin and A. Strauss, Basics of qualitative research: Techniques and procedures for developing grounded theory. SAGE Publications, Incorporated, 2015.

[5] K. Charmaz, "Grounded theory as an emergent method," in Handbook of emergent methods, 2008, pp. 155-172.

[6] G. A. Bowen, "Grounded theory and sensitizing concepts," Int. J. Qual. Methods, vol. 5, no. 3, pp. 12-23, 2006. 
[7] K. Charmaz, "Grounded theory: Objectivist and constructivist methods," in Strategies for qualitative inquiry, 2nd ed., N. K. Denzin and Y. S. Lincoln, Eds. 2003, pp. 249-291.

[8] J. W. Creswell, Research Design: Qualitative, Quantitative, and Mixed Methods Approaches. Thousand Oaks, CA: SAGE Publications, 2003.

[9] D. L. Morgan, "Paradigms lost and pragmatism regained: Methodological implications of combining qualitative and quantitative methods," J. Mix. Methods Res., vol. 1, no. 1, pp. 48-76, 2007.

[10] P. Shannon-Baker, “'But I wanted to appear happy': How using arts-informed and mixed methods approaches complicate qualitatively driven research on culture shock," Int. J. Qual. Methods, vol. 14, no. 2, pp. 34-52, 2015.

[11] C. McCall and C. Edwards, "New Perspectives for Implementing Grounded Theory," Stud. Eng. Educ., vol. 1, no. 2, 2021.

[12] Morgan, D. L. (2020). Pragmatism as the basis for grounded theory. The Qualitative Report, 25 (1), 64-73.

[13] J. M. Corbin and A. Strauss, "Grounded theory research: Procedures, canons, and evaluative criteria," Qual. Sociol., vol. 13, no. 1, pp. 3-21, 1990.

[14] J. W. Creswell, Qualitative inquiry and research design. SAGE Publications, 2013.

[15] "Best Engineering Schools Ranked in 2022 - US News Rankings." https://www.usnews.com/best-graduate-schools/top-engineering-schools/eng-rankings (accessed Apr. 19, 2021).

[16] E. R. Babbie, The basics of social research, International Student Edition. USA: Thomas Wadworth, 2008.

[17] S. Bhaduri and T. Roy, "A word-space visualization approach to study college of engineering mission statements," presented at the Frontiers in Education Conference, Indianapolis, IN, 2017.

[18] K. Charmaz, Constructing Grounded Theory: A Practical Guide Through Qualitative Analysis. SAGE, 2006.

[19] S. L. Morrow and M. L. Smith, "Constructions of survival and coping by women who have survived childhood sexual abuse.," J. Couns. Psychol., vol. 42, no. 1, p. 24, 1995.

[20] G. Glaser Barney, Theoretical sensitivity: advances in the methodology of grounded theory. Mill Valley, CA: The Sociology Press, 1978. 